\title{
Pulmonary complications after cardiac surgery: breathing down the patient's neck
}

\author{
P.L. Temporelli1, M. Ambrosetti2
}

Monaldi Arch Chest Dis 2011; 75: 2, 109-111.

Keywords: Cardiac surgery, Rehabilitation, Chest physiotherapy.

${ }^{1}$ Division of Cardiology, IRCCS Salvatore Maugeri Foundation, Veruno (NO), Italy. President of the Gruppo Italiano di Cardiologia Riabilitativa - Italian Association for Cardiovascular Prevention, Rehabilitation and Epidemiology (GICR-IACPR).

2 Cardiovascular Rehabilitation Unit, "Le Terrazze" Clinic, Cunardo (VA), Italy. Gruppo Italiano di Cardiologia Riabilitativa Italian Association for Cardiovascular Prevention, Rehabilitation and Epidemiology (GICR-IACPR) Board.

Correspondence: Dr. Pier Luigi Temporelli, Divisione di Cardiologia Riabilitativa, Fondazione Salvatore Maugeri, IRCCS, Istituto Scientifico di Veruno, Via per Revislate 13, 28010 Veruno (NO), Italy; e-mail: pierluigi.temporelli@fsm.it; presidente@iacpr.it

In Italy more than 50,000 cardiac interventions are currently performed every year, $40 \%$ of which require valve implantation. The clinical characteristics of patients who undergo cardiac surgery have changed radically over the last decade: due to advances in perioperative care, older and moderateto-severe risk patients are now more frequently being referred to cardiac surgery, highlighting a need for increased improvement and stabilisation of surgical and medical issues. Structured rehabilitation, mainly offered through inpatient programmes, is now recognized as a standard form of care for cardiac surgery patients. Historically, the primary role of rehabilitation after cardiac surgery is to treat disability and improve functional capacity. Exercise training after heart valve replacement increases peak $\mathrm{VO}_{2}$, cardiovascular response at submaximal workloads, and quality of life with higher rates of returning to work [1], while in patients following coronary revascularisation it is known to reduce cardiovascular events as well as hospital readmissions [2].

In order to achieve better rehabilitation outcomes, patients' symptoms and needs after cardiac surgery must be comprehensively addressed. Apart from functional recovery and cardiovascular risk reduction, a multidisciplinary integrated team approach can also positively affect post-operative pain, complications at surgical site, cardioprotective drug titration, and lifestyle modifications. Importantly, comorbidities markedly affect patients' health status after cardiac surgery, and require adequate attention.

In the current issue of Monaldi Archives for Chest Disease - Pulmonary series, Westerdahl and Olsén [3] carried out a national survey in Sweden aimed at establishing the current practice of chest physiotherapy for patients undergoing cardiac surgery. Through a questionnaire sent to about thirty physiotherapists working in eight departments of cardiothoracic surgery, the authors obtained information on the use of breathing exercises after cardiac surgery and found that: i) advice for post-operative breathing exercise is regularly prescribed in Sweden, ii) the two most frequently used techniques are deep breathing with or without a PEP device, and iii) there is still a considerable heterogeneity in treatment options and timing of intervention.

The strengths of the study include the national coverage of the survey (almost all Swedish cardiac surgery units were represented), and the well-designed questionnaire (suitable for future comparison among different institutions and different countries). Among the study limitations is the short time horizon of the survey, i.e. the evaluation of chest physiotherapy was limited to the first five post-operative days and did not include other interventions offered during a structured cardiac rehabilitation programme. A second limitation is the exclusion of patients with complicated open-heart surgery due to neurological, circulatory or ventilation-related conditions; such patients are now becoming more common as an effect of the increased age and comorbidity of "clear for surgery" subjects.

Conceptually, a strong rationale for chest physiotherapy after cardiac surgery exists. In addition to the intervention per se, thoracotomy and general anesthesia contribute to reducing diaphragm excursion, lung volumes and functional capacity, to the point even of inducing a ventilation/perfusion mismatch and bronchial stasis. In addition to major pulmonary complications such as pneumonia, pulmonary embolism and respiratory distress, cardiac surgery is also associated with increased risk of lung atelectasis, pleural effusion, and paralysis of the frenic nerve. Both obstructive and restrictive pulmonary impairment may develop as a consequence of cardiac surgery, and conventional chest physical therapy (whose main goals are to improve mucus clearance and pulmonary function) can help prevent this event.

Unfortunately, there is not a wide consensus about the most appropriate program for this patient population. A still unsolved question is the role of incentive spirometry in preventing pulmonary complications after cardiac surgery: a recent 
Cochrane intervention review [4] found evidence from four small trials that incentive spirometry offers no advantage over standard physical therapy either in preventing breathing complications (mainly atelectasis and pneumonia) or in limiting the negative effects of surgery on pulmonary function. However, these results should be interpreted cautiously in view of the small number of patients evaluated. Westerdahl and Olsén [3] in their work found a high prescription rate of breathing exercises and coughing/huffing techniques, while other less conventional treatments were prescribed in less than $10 \%$ of cases in the immediately post-operative period. A second question is whether presurgery respiratory physiotherapy may reduce the incidence of post-surgery breathing complications. In a systematic review of 18 trials performed by Pasquina et al. in 2003 [5], the usefulness of prophylactic chest physiotherapy was tested for several end points (atelectasis, incidence of pneumonia, partial pressure of arterial oxygen, vital capacity, and forced expiratory volume in one second), and did not find a clear confirmation. By contrast, more recent controlled trials documented a lower incidence of atelectasis among patients treated with a combined approach involving incentive spirometry, deep breathing exercise, coughing and early ambulation [6].

Pulmonary complications are now recognised as important comorbidities in patients who have undergone both coronary artery bypass surgery and heart valve repair or replacement, also in terms of event-related complications or chronic illnesses. In the years 2008 and 2009, the Italian Association for Cardiovascular Prevention, Rehabilitation, and Epidemiology (IACPR-GICR) carried out two large prospective, longitudinal, multicentric surveys providing clinical information on patients completing cardiac rehabilitation in Italy, with the acronyms of ISYDE (Italian SurveY on carDiac rEhabilitation) and ICAROS (Italian survey on CArdiac RehabilitatiOn and Secondary prevention after cardiac revascularization). Globally, the two studies enrolled approximately 3,500 patients admitted to $165 \mathrm{CR}$ units, representing more than $85 \%$ of all 190 registered facilities in Italy. The studies utilized a web-based clinical record (e$\mathrm{CRF}$ ) compiled on the institutional website (www.iacpr.it), and data quality was also improved due to the availability of online guides and a helpdesk. Information regarding index events, complications during the acute phase, comorbidities, the rehabilitation programme, lifestyle, and therapies were collected. Armed with ISYDE and ICAROS pictures of "real-life" patients [7-8], health operators should now focus particular attention on pulmonary-related situations and unmet clinical needs after cardiac surgery. Data from these surveys has shown that, prior to cardiac rehabilitation admission, about $5 \%$ of patients had mechanical ventilation in the perioperative in-hospital stay, and about $4 \%$ of patients were treated for post-operative pleural effusion by means of thoracenthesis. During the rehabilitation phase, another $5 \%$ of patients required non-invasive me- chanical ventilation. Despite the low proportion of patients showing acute respiratory distress during hospitalisation in cardiac surgery units, the presence of chronic obstructive pulmonary disease (COPD) as a comorbidity was associated with an increased number of intervention/procedures or prolonged duration of the rehabilitation programme in up to $13 \%$ of patients. This effect size was superior to other well-known and feared conditions in the cardiac rehabilitation setting (such as heart failure or renal impairment), and only just superceded by the presence of other peripheral localizations of atherosclerosis. In a combined analysis of 2,256 ISYDE and ICAROS cardiac surgery patients [9], COPD was significantly associated with the occurrence of atrial fibrillation during the cardiac rehabilitation phase, and affected up to $20 \%$ of patients who experienced de-novo or recurrent episodes of atrial fibrillation, in addition to arrhythmias that developed during the acute phase. Since atrial fibrillation is a well-known condition potentially worsening the cardiovascular prognosis after cardiac surgery, it could be argued that treating appropriately respiratory impairments in the post-operative phase (also with physical techniques) may improve the global outcome of these patients.

Thus, pulmonary complications frequently affect the course of cardiac surgery patients and are responsible for increased morbidity, mortality, and healthcare costs. Respiratory physiotherapy after cardiac surgery is widely used, but unfortunately strong evidence for its benefit and documenting the optimal combination and frequency of different techniques is still lacking. Despite this, the pulmonary status in this patient population should be routinely assessed, with a view to ameliorating respiratory symptoms and specific causes of disability, whatever the method of chest physiotherapy preferred at local level.

\section{References}

1. Williams MA, Ades PA, Hamm LF, et al. Clinical evidence for a health benefit from cardiac rehabilitation: an update. Am Heart J 2006; 152: 835-841.

2. Hedback B, Perk J, Hornblad M, et al. Cardiac rehabilitation after coronary artery bypass surgery: 10-year results on mortality, morbidity and readmissions to hospital. J Cardiovasc Risk 2001; 8: 153-158.

3. Westerdahl E, Fagevik Olsén M. Chest physiotherapy and breathing exercises for cardiac surgery patients in Sweden - A National survey of practice. Monaldi Arch Chest Dis 2011; 75: 112-119.

4. Freitas ERF, Soares B, Cardoso JR, Atallah AN. Incentive spirometry for preventing pulmonary complications after coronary artery bypass graft. Cochrane Database of Systematic Reviews 2007, Issue 3. Art. No.: CD004466. DOI: 10.1002/14651858.CD004466.pub2.

5. Pasquina $P$, Tramèr MR, Walder B. Prophylactic respiratory physiotherapy after cardiac surgery: systematic review. BMJ 327 (7428): 1379.

6. Yanez-Brage I, Pita-Fernandez S, Juffé-Stein A, Martinez-Gonzalez U, Pertega-Diaz S, Mauleon-Garcia A. Respiratory physiotherapy and incidence of pulmonary complications in off-pump coronary artery bypass graft surgery: an observational follow-up study. BMC Pulm Med 2009; 9: 36-46. 
7. Tramarin R, Ambrosetti M, De Feo S, Piepoli M, Riccio C, Griffo R; ISYDE-2008 Investigators of the Italian Association for Cardiovascular Prevention, Rehabilitation and Prevention. The Italian Survey on Cardiac Rehabilitation-2008 (ISYDE-2008). Part 3. National availability and organization of cardiac rehabilitation facilities. Official report of the Italian Association for Cardiovascular Prevention, Rehabilitation and Epidemiology (IACPR-GICR). Monaldi Arch Chest Dis 2008; 70: 175-205.

8. Griffo R, Fattirolli F, Temporelli PL, Tramarin R. Italian survey on cardiac rehabilitation and secondary pre- vention after cardiac revascularization: ICAROS study. A survey from the Italian cardiac rehabilitation network: rationale and design. Monaldi Arch Chest Dis 2008; 70: 99-104, 106.

9. Ambrosetti M, Tramarin R, Griffo R, De Feo S, Fattirolli F, Vestri A, Riccio C, Temporelli PL; on behalf of the ISYDE and ICAROS investigators of the Italian Society for Cardiovascular Prevention, Rehabilitation and Epidemiology (IACPR-GICR). Late postoperative atrial fibrillation after cardiac surgery: a national survey within the cardiac rehabilitation setting. J Cardiovasc Med 2011; 12: 390-395.

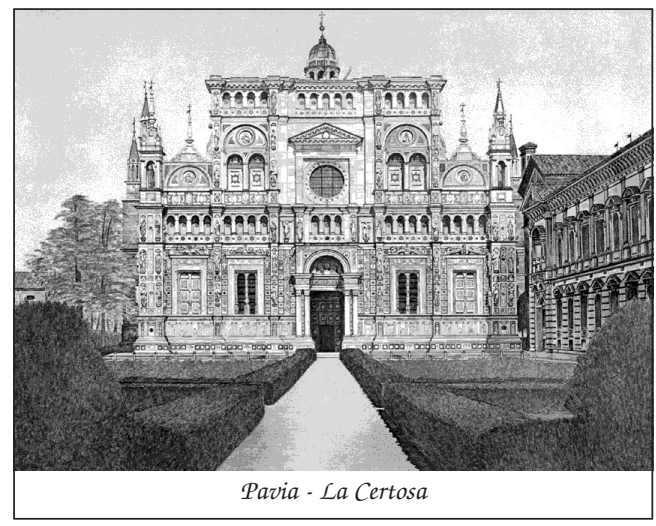

